\title{
Spatial variability and its scale dependency of observed and modeled soil moisture over different climate regions
}

\author{
B. $\mathbf{L i}^{1,2}$ and M. Rodell ${ }^{2}$ \\ ${ }^{1}$ Earth System Science Interdisciplinary Center, University of Maryland, College Park, Maryland 20740, USA \\ ${ }^{2}$ Hydrological Sciences Laboratory, NASA Goddard Space Flight Center, Greenbelt, Maryland 20771, USA
}

Correspondence to: B. Li (bailing.li@nasa.gov)

Received: 17 August 2012 - Published in Hydrol. Earth Syst. Sci. Discuss.: 7 September 2012

Revised: 28 January 2013 - Accepted: 2 March 2013 - Published: 19 March 2013

\begin{abstract}
Past studies on soil moisture spatial variability have been mainly conducted at catchment scales where soil moisture is often sampled over a short time period; as a result, the observed soil moisture often exhibited smaller dynamic ranges, which prevented the complete revelation of soil moisture spatial variability as a function of mean soil moisture. In this study, spatial statistics (mean, spatial variability and skewness) of in situ soil moisture, modeled and satellite-retrieved soil moisture obtained in a warm season (198 days) were examined over three large climate regions in the US. The study found that spatial moments of in situ measurements strongly depend on climates, with distinct mean, spatial variability and skewness observed in each climate zone. In addition, an upward convex shape, which was revealed in several smaller scale studies, was observed for the relationship between spatial variability of in situ soil moisture and its spatial mean when statistics from dry, intermediate, and wet climates were combined. This upward convex shape was vaguely or partially observable in modeled and satellite-retrieved soil moisture estimates due to their smaller dynamic ranges. Despite different environmental controls on large-scale soil moisture spatial variability, the correlation between spatial variability and mean soil moisture remained similar to that observed at small scales, which is attributed to the boundedness of soil moisture. From the smaller support (effective area or volume represented by a measurement or estimate) to larger ones, soil moisture spatial variability decreased in each climate region. The scale dependency of spatial variability all followed the power law, but data with large supports showed stronger scale dependency than those with smaller supports. The scale dependency of soil moisture variability also varied with climates, which may be linked to
\end{abstract}

the scale dependency of precipitation spatial variability. Influences of environmental controls on soil moisture spatial variability at large scales are discussed. The results of this study should be useful for diagnosing large scale soil moisture estimates and for improving the estimation of land surface processes.

\section{Introduction}

Spatial variability of soil moisture plays an important role in the estimation of land surface fluxes (evapo-transpiration (ET) and runoff), due to the non-linear relationship between soil moisture and the associated physical processes. Characterizing soil moisture spatial variability is also important for validating satellite-derived soil moisture, as the number of soil samples needed to accurately represent the spatial mean within the footprint of satellites depends on soil moisture spatial variability (Brocca et al., 2012; Famiglietti et al., 2008; Wang et al., 2008). In addition, since the footprint of satellite-derived soil moisture is often larger than the resolution of many hydrological applications, downscaling retrieved soil moisture is often necessary, which can be helped with knowledge of soil moisture spatial variability and its scale dependency.

Many field experiments have been conducted at catchment scales to measure soil moisture in high spatial density and to examine the spatial variability of soil moisture. One important aspect of soil moisture spatial variability that has been repeatedly investigated is its dependency on spatial mean soil moisture. Various conclusions have been reached about this relationship (see reviews by Choi et al., 2007; Ryu and 
Famiglietti, 2005; Famiglietti et al., 1999, 2008): some studies have indicated that spatial variability increased as mean soil moisture became wetter while others have indicated just the opposite. Several hypotheses have been explored as to how the two opposite correlations could co-exist (Crow and Wood, 1999; Rodriguez-Iturbe et al., 1995). Famiglietti et al. (2008), Brocca et al. (2012) and Rosenbaum et al. (2012) showed an upward convex (or concave) relationship that consists of both negative and positive correlations. A noticeable shortcoming of past studies is that they are based on data collected in a short time period and/or in specific climate regions (most with intermediate precipitation), which limited the dynamic range of soil moisture and prevented the full revelation of this relationship.

The theoretical foundation for scaling spatial variability is the power law, which states that the spatial variability at one scale is related to that at another scale by the power of a scaling factor (Crow and Wood, 2002; Hu et al., 1998). Studies using spatial aggregations have shown that the relationship between soil moisture variability and its spatial resolution generally follows the power law (Crow and Wood, 2002; Hu et al., 1998; Parada and Liang, 2003). Famiglietti et al. (2008) and Brocca et al. (2012) examined another aspect of the power law using field measurements, that is, the relationship between spatial variability and the extent (the maximum spatial range of measurements) and showed that the variability of soil moisture increased as the extent $(<50 \mathrm{~km})$ increased. There is no knowledge about the scale dependency of spatial variability at even larger extents, which can be important for understanding the scale dependency of large-scale land surface processes that interact with soil moisture.

In addition to in situ measurements, models provide spatially and temporally continuous soil moisture estimates with global coverage. Model estimates are derived based on largescale representations of static parameters and meteorological forcing fields and inevitably impacted by imperfect model physics and parameters. Satellite-derived soil moisture estimates also provide global coverage but are influenced by errors in retrieval procedures and limitations of the sensor (Jackson, 1993; Njoku et al., 2003), in addition to their larger spatial representations. Studying the spatial variability of these two data sources along with in situ data should provide additional insights into the nature of soil moisture spatial variability and its scale dependency, as well as useful information for improving model estimated soil moisture.

The objective of this study is to examine the spatial variability of in situ, modeled and remotely sensed soil moisture at large extents $(>100 \mathrm{~km})$ over different climate regions and their scale dependency. The three types of data are: in situ soil moisture measurements from the Soil Climate Analysis Network (SCAN, Schaefer et al., 2007), North American Land Data Assimilation System (NLDAS) Noah model estimates, and Advanced Microwave Scanning RadiometerEOS (AMSR-E) soil moisture retrievals. The SCAN network, which has stations across the US, provides diverse climate conditions to study the impact of climate on the spatial variability of soil moisture in a more complete soil moisture range than examined in previous studies.

In the rest of the paper, the scale triplet, spacing (the distance between two sampling locations), support (the effective area or volume represented by each data type) and extent (maximum spatial distance covering all sampling points), as defined by Western and Blösch (1999), is used to discuss the scale dependency of soil moisture spatial variability.

\section{Data and study design}

Figure 1 shows the location of the 109 SCAN sites used for this study. To study the relationship between soil moisture variability and climate, the continental US was split into three regions along the $-104^{\circ}$ and $-96^{\circ}$ longitude lines: West, Mid-continent (MidCon) and East, which roughly represent dry (noting that no SCAN sites exist on West Coast), intermediate and wet conditions, respectively. In West and East, two sub-regions were further chosen to create a smaller extent for statistical analysis. The sub-region in West essentially encompasses the state of Utah (thereafter referred to as Utah) and the sub-region in East is located in the Mississippi/Tennessee/Alabama area (referred to as MissTenn). No sub-region was selected for MidCon due to lack of densely located SCAN stations in the region. The number of SCAN locations in each region is given in Table 1 . The extent is about 500 to $700 \mathrm{~km}$ for the sub-regions and about 2000 to $3000 \mathrm{~km}$ for the three large climate regions. Figure 1 also shows three series of concentric squares, with side lengths ranging from 110 to $1500 \mathrm{~km}$, which were used to study the scale dependency of modeled and remotely sensed soil moisture in Sect. 4.4.

Most SCAN stations began measuring soil moisture in the early 1990s, while new stations such as those in Utah were added in 2007. To include as many stations as possible, 2008 was chosen as the study year, which was further limited to 1 May-15 November (198 days) to eliminate the impact of freezing/thawing conditions on the analysis. SCAN soil moisture is recorded hourly at the 5, 10, 20, 50 and $100 \mathrm{~cm}$ depths using Hydro Probe, which converts soil dielectric permittivity to water content through calibrated equations (Seyfried et al., 2005). As SCAN stations generally are located in agricultural areas, typical soil types are silt loam and fine sandy loam.

Modeled soil moisture fields were generated by the Noah land surface model embedded in NLDAS (Mitchell et al., 2004). Noah has been developed and maintained by NOAA's Environment Modeling Center for use in their coupled weather forecasting system. The soil moisture simulation of Noah is based on a vertical discretization of the Richards' equation into four soil layers with thicknesses of 10, 30, 60, and $100 \mathrm{~cm}$. Noah was never calibrated against SCAN soil moisture and thus its estimates are independent of the in 
Table 1. The number of SCAN stations, temporally averaged (over 198 days) spatial mean and spatial variability (StD) of soil moisture $\left(\mathrm{cm}^{3} \mathrm{~cm}^{-3}\right)$ in each region. Statistics were calculated using data values at SCAN sites, except the numbers in parentheses, which were computed using all grid points in each region.

\begin{tabular}{|c|c|c|c|c|c|c|c|}
\hline \multirow[b]{2}{*}{ Region } & \multirow{2}{*}{$\begin{array}{l}\text { Number of } \\
\text { SCAN sites } \\
\text { (NLDAS } \\
\text { grid points) }\end{array}$} & \multicolumn{2}{|c|}{ SCAN } & \multicolumn{2}{|c|}{ NLDAS } & \multicolumn{2}{|c|}{ AMSR-E } \\
\hline & & mean & StD & mean & StD & mean & StD \\
\hline West & $34(25152)$ & 0.11 & 0.082 & $0.17(0.18)$ & $0.051(0.069)$ & 0.06 & 0.024 \\
\hline Utah & $16(1681)$ & 0.09 & 0.072 & $0.17(0.17)$ & $0.042(0.045)$ & 0.06 & 0.020 \\
\hline MidCon & 19 (15168) & 0.19 & 0.112 & $0.21(0.22)$ & $0.074(0.060)$ & 0.12 & 0.050 \\
\hline East & $56(35520)$ & 0.23 & 0.109 & $0.25(0.24)$ & $0.046(0.050)$ & 0.19 & 0.045 \\
\hline Miss-Tenn & $32(3185)$ & 0.25 & 0.106 & $0.26(0.25)$ & $0.038(0.037)$ & 0.19 & 0.039 \\
\hline
\end{tabular}

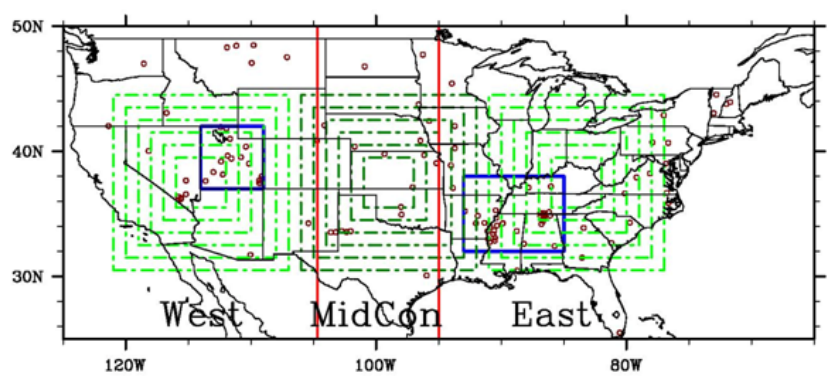

Fig. 1. SCAN site locations (in brown circles), climate regions (divided by red lines), sub-regions (in blue rectangles), and three series of concentric squares (in dark and light green) used in the scaledependency study for Noah and AMSR-E soil moisture data.

situ measurements. NLDAS precipitation is based on daily measurements from over 10000 gauges located in the US, which are then temporally disaggregated into hourly data using hourly Doppler radar images (Cosgrove et al., 2003). As given in Table 2, NLDAS precipitation (for the study period) generally agrees with gauged data recorded at SCAN sites, especially in terms of capturing climate differences in each region. For NLDAS, total precipitation averaged over all grid points in each region is also provided in Table 2, which shows some differences from those averaged over SCAN locations only, due to the scattered nature of the SCAN network. The 1-km STATSGO soil texture used by NLDAS/Noah was also found (not shown) in good agreement with field soil descriptions at SCAN sites. Hourly Noah soil moisture estimates, which are integrated on a 0.125 degree grid were extracted from archived NLDAS/Noah outputs (http://www.emc.ncep.noaa.gov/mmb/nldas/).

Advanced Microwave Scanning Radiometer for EOS (AMSR-E) soil moisture retrievals produced by the NOAA's National Environmental Satellite, Data and Information Service (Zhan et al., 2008) were used in this study. This AMSR$\mathrm{E}$ product, derived from the $\mathrm{X}$-band frequency brightness temperature using the Single Channel Retrieval algorithm (Jackson, 1993), has larger dynamic ranges than the official
Table 2. Total SCAN and NLDAS precipitation (mm) for the study period (1 May-15 November).

\begin{tabular}{lrrr}
\hline Region & SCAN & $\begin{array}{r}\text { NLDAS } \\
\text { (at SCAN sites) }\end{array}$ & $\begin{array}{r}\text { NLDAS } \\
\text { (at all grid points) }\end{array}$ \\
\hline West & 181 & 173 & 222 \\
Utah & 134 & 142 & 132 \\
MidCon & 486 & 554 & 483 \\
East & 580 & 702 & 670 \\
Miss-Tenn & 596 & 673 & 661 \\
\hline
\end{tabular}

AMSR-E product (Njoku et al., 2003) with more realistic mean values in wetter climates than the official product (not shown). The sensing depth of the AMSR instrument is believed to be about $1-2 \mathrm{~cm}$ from the surface (Njoku et al., 2003). AMSR-E retrievals, with a 25 by $25 \mathrm{~km}$ spatial resolution and ca. 1-2 retrievals per day, have the largest support among the three data types.

\section{Statistical moments}

To analyze the three data sets, the first three statistical moments were calculated for daily soil moisture values in each climate region and sub-region. All statistics were calculated using NCL (http://www.ncl.ucar.edu/overview.shtml) buildin functions and their mathematical formulations are provided here. For $N$ soil moisture values on day $t$ in any given region, their spatial mean, $M$, is given by

$M_{t}=\frac{1}{N} \sum_{i=1}^{N} \theta_{i, t}$,

where $\theta_{i, t}$ is the soil moisture at location $i$ on day $t$.

Following the same notation, the spatial variability of soil moisture is measured by the sample standard deviation,

$\sigma_{t}=\left(\frac{1}{N-1} \sum_{i=1}^{N}\left(\theta_{i, t}-M_{t}\right)^{2}\right)^{1 / 2}$, 
and the skewness, which measures the asymmetry of data distribution, is defined as

$$
S_{t}=\frac{\left(\sum_{i=1}^{N}\left(\theta_{i, t}-M_{t}\right)^{3}\right) / N}{\left(\left[\sum_{i=1}^{N}\left(\theta_{i, t}-M_{t}\right)^{2}\right] / N\right)^{3 / 2}} .
$$

For two soil moisture time series at any given location (i), their temporal correlation is given by the Pearson correlation coefficient:

$r_{i}^{12}=\frac{1}{N_{t} \sigma_{i}^{1} \sigma_{i}^{2}} \sum_{t=1}^{N_{t}}\left(\theta_{i, t}^{1}-Y_{i}^{1}\right)\left(\theta_{i, t}^{2}-Y_{i}^{2}\right)$,

where superscripts 1 and 2 represent the two time series; $N_{t}$ is the number of data points in the time series, which is 198 days for this study; $Y_{i}$ and $\sigma_{i}$ are the temporal mean and standard deviation at location $i$, respectively.

\section{Results}

\subsection{Mean, spatial variability and skewness}

Daily soil moisture of SCAN, Noah and AMSR-E was first calculated at each SCAN location and then used to compute spatial statistics - mean, variability and skewness - in each region (climate and sub-region) for each data set. Because AMSR-E retrievals are surface observations only, the statistical analysis was limited to the SCAN measurements at the 5$\mathrm{cm}$ depth and the top layer of Noah estimates. All soil moisture values used for statistical calculations and presented in the graphs are in volumetric water content $\left(\mathrm{cm}^{3} \mathrm{~cm}^{-3}\right)$.

Figure 2 is the box plot of the daily mean soil moisture for the three data types in each region. The lower, center and upper limits of each box represent the 25th, 50th and 75th percentiles of spatial means while the two whiskers represent the minimum and maximum value in each data set. All three data types show sensitivity to climate conditions with median soil moisture increasing from the West to the East. Observed soil moisture (SCAN and AMSR-E) is more sensitive to changes in climate conditions than Noah, whose median soil moisture increases less than the others as the climate becomes wetter.

Noah and AMSR-E estimates have smaller dynamic ranges in all regions, as the boxes are generally smaller than those of SCAN. In West and Utah, Noah estimates show positive bias relative to SCAN, with the median value near 0.2. AMSR-E retrievals, on the other hand, generally exhibit a drier bias in each region. Temporally averaged (over the 198 days) daily mean values given in Table 1 further confirm these biases. Many factors could contribute to these discrepancies in spatial means. AMSR-E soil moisture data were affected by the shallow sensing depth $(1-2 \mathrm{~cm})$ and lack of retrievals during rainfall, which may have reduced their ability to capture the seasonal variability seen in the SCAN and

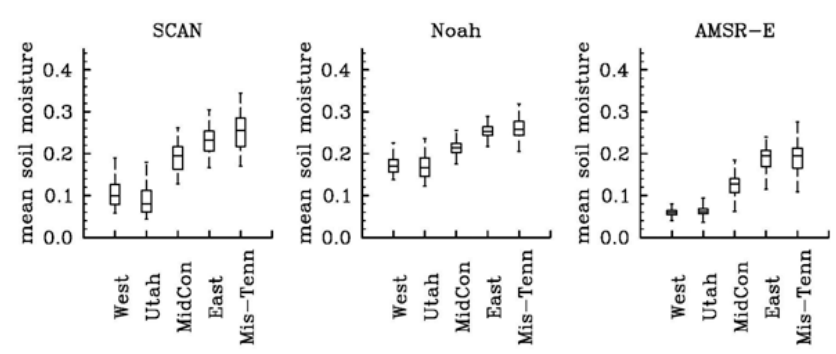

Fig. 2. Box plot of daily mean soil moisture $\left(\mathrm{cm}^{3} \mathrm{~cm}^{-3}\right)$ in climate regions and sub-regions for the three data types. Statistics of Noah and AMSR-E were calculated using data at SCAN locations.

Noah soil moisture datasets. The reduced sensitivity of Xband brightness temperature to soil moisture in the growing season may also prevent accurate retrievals (Jackson, 1993). Similarly, Noah soil moisture is affected by inaccurate model parameters (including dynamic forcing fields) and deficiencies in model physics. Both Noah and AMSR-E soil moisture are also averaged values over an area where the vegetation cover, soil texture and the atmospheric forcing may differ from those at the location of SCAN sites. Exploring the exact cause for such deviations is beyond the scope of this study; instead, the rest of this study focuses on how mean soil moisture, in particular, its magnitude relative to the mid-range soil moisture (defined as the arithmetic mean of the maximum and minimum soil moisture), affects the higher moments of soil moisture. The soil moisture range for AMSR-E is $0-0.5$ and about $0-0.45$ for SCAN data, based on the tested soil types (Seyfried et al., 2005). Noah soil moisture is limited by the wilting point and porosity, which are 0.07 and 0.44 , respectively, based on the averaged values of all soil types appeared in the study area. Given the limited information on SCAN soil properties, the mid-range soil moisture is approximately at 0.2 for SCAN and 0.25 for Noah and AMSR-E.

Figure 3 shows the standard deviation of daily soil moisture as a function of spatial means. For SCAN soil moisture, statistics from all regions collectively suggested an upward convex relationship. In West and Utah, soil moisture variability increases as soils become wetter, i.e., positive correlation, while the opposite is observed in East and Miss-Tenn (more noticeable in Miss-Tenn). The spatial variability peaks in MidCon where no obvious trend is observed. This upward convexity was observed in some previous studies in smaller scales (e.g., Famiglietti et al., 2008; Brocca et al., 2007, 2012; Rosenbaum et al., 2012) but has not been observed at the continental scale. Comparing SCAN statistics in Figs. 2 and 3 reveals that the upward convexity is directly linked to the overall soil wetness: when mean soil moisture is below its mid-range (as in West and Utah), soil moisture variability increases with soil wetness; when mean soil moisture is above 0.2 (in East and Miss-Tenn), the variability decreases with increased wetness. In MidCon where mean soil moisture values are centered at 0.2, no trend is observed, and overall 

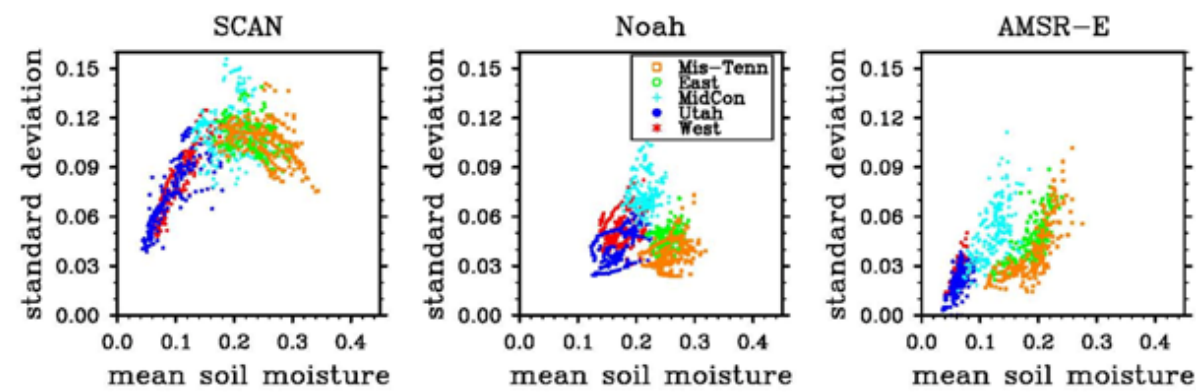

Fig. 3. Spatial variability (standard deviation) of soil moisture as a function of mean soil moisture $\left(\mathrm{cm}^{3} \mathrm{~cm}^{-3}\right)$ for the three data types. Statistics of Noah and AMSR-E were calculated using data at SCAN locations.

variability is the highest. This transitioning of correlation from the positive to negative around the mid-range soil moisture was also noted by Penn et al. (2009) and Rosenbaum et al. (2012). Rosenbaum et al. (2012) also showed a more substantial decreasing trend in the wetter range of soil moisture, which is missing in East because mean soil moisture in the region did not reach the wetter end.

Spatial variability of Noah soil moisture shows positive correlation with mean soil moisture in West and Utah and no correlation in other regions. The lack of negative correlation in East/Miss-Tenn is associated with the fact that the spatial means of these two regions are very close to the midrange soil moisture. As SCAN, Noah soil moisture exhibits the largest spatial variability in MidCon. The spatial variability of AMSR-E soil moisture, on the other hand, shows positive correlation with mean soil moisture in all regions. This behavior is also linked to the fact that the median mean of AMSR-E retrievals is always near or below its mid-range soil moisture (0.25) in each region (Fig. 2). More discussions on the contributing factors to this upward convexity will be given in Sect. 4.3.

To illustrate the temporal variation of spatial variability, Fig. 4 shows the time series of spatial mean soil moisture, soil moisture spatial variability (StD) and mean precipitation in the three climate regions. SCAN soil moisture shows strong seasonality with larger dynamic ranges. Noah soil moisture exhibits similar seasonality but with smaller dynamic ranges and noticeable wetter conditions in the summer. Both of them also show strong correlation with daily precipitation. No similar seasonality is observed in AMSR-E soil moisture, which may be attributed to its shallow sensing depth and reduced sensitivity of brightness temperatures to moisture content in the growing season when vegetation water content is high (Jackson, 1993).

Temporal correlations and root mean square errors (RMSE) of the Noah and AMSR-E estimates with respect to SCAN soil moisture at each SCAN site were also calculated and their region-averaged values are given in Table 3. Noah shows better correlation and lower RMSE than AMSR-E in all regions, except in West where AMSR-E has lower RMSE. As can be seen from Fig. 4, the low correlation of AMSR-E
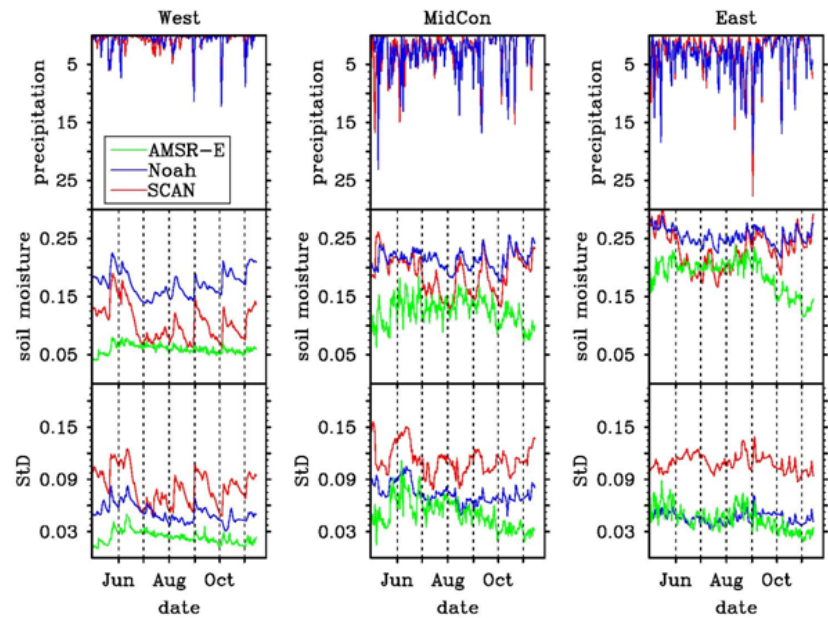

Fig. 4. Time series of daily (averaged over values at SCAN locations) precipitation $(\mathrm{mm})$, spatial mean soil moisture $\left(\mathrm{cm}^{3} \mathrm{~cm}^{-3}\right)$ and soil moisture standard deviation $(\mathrm{StD})$ in climate regions. Statistics of Noah and AMSR-E were calculated using data at SCAN locations.

with SCAN measurements mainly stems from its lack of seasonality and no correlation with daily precipitation. As discussed earlier, many factors contributed to the difference between AMSR-E/Noah and SCAN soil moisture including the scattered nature of SCAN sites in each region. Thus, these statistics are provided for quantifying the difference between the two data sets and for the purpose of discussing their impacts on spatial variability and are not presented as evaluations of Noah and AMSR-E soil moisture.

The temporal variation of spatial variability in Fig. 4 follows the correlation between spatial variability and mean soil moisture as observed in Fig. 3. For instance, spatial variability increases/decreases as mean soil moisture increases/decreases in West for all three data sets. The spatial variability of Noah and AMSR-E generally varies less than that of SCAN, resulting from their smaller dynamic ranges. While the dynamic range of mean soil moisture for SCAN data remains more or less the same across all regions, the dynamic range of its spatial variability decreases from West 

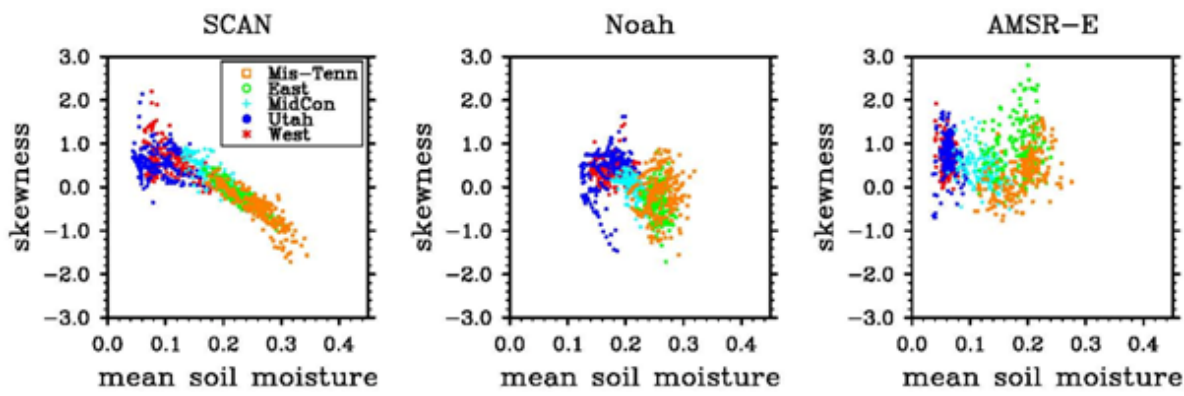

Fig. 5. Soil moisture skewness as a function of spatial mean soil moisture $\left(\mathrm{cm}^{3} \mathrm{~cm}^{-3}\right)$ for the three data types. Statistics of Noah and AMSR-E were calculated using data at SCAN locations.

Table 3. Temporal correlations $(r)$ and root mean square errors (RMSE) of Noah/AMSR-E soil moisture with respect to SCAN measurements. Statistics were first calculated at each SCAN site and then averaged over all SCAN locations in each region.

\begin{tabular}{lrrrrrr}
\hline & \multicolumn{2}{c}{ Noah } & & \multicolumn{2}{c}{ AMSR-E } \\
\cline { 2 - 3 } \cline { 5 - 6 } Region & $r$ & RMSE & & $r$ & RMSE \\
\hline West & 0.61 & 0.104 & & 0.31 & 0.08 \\
MidCon & 0.66 & 0.087 & & 0.42 & 0.11 \\
East & 0.64 & 0.090 & & 0.19 & 0.11 \\
\hline
\end{tabular}

to East. This is due to the fact that soil moisture in MidCon and East fluctuates around its mid-range soil moisture and therefore any increasing or decreasing trend of spatial variability is frequently reversed. Similar behaviors are seen with Noah estimates, but not with AMSR-E retrievals. These results demonstrate that soil moisture spatial variability is sensitive to mean soil moisture, especially its relative magnitude with respect to the mid-range soil moisture, and its temporal variability.

Skewness measures the asymmetry of a probability distribution and is important for ensemble-related data assimilation techniques, which often assume normality. Figure 5 shows the skewness of daily soil moisture for the three data types. SCAN exhibits climate dependent skewness: soil moisture is positively skewed in West and Utah, negatively skewed in East and Miss-Tenn, and centered at zeroskewness in MidCon. These behaviors can be explained using the boundedness of soil moisture. For example, in the dry climate where median soil moisture value is smaller than the mid-range soil moisture, the left tail (representing values below the median) of soil moisture distributions is suppressed by the zero bound, which leads to positive skewness.

Noah estimates exhibit a somewhat similar behavior in skewness across different climate zones. Because their mean values do not reach very dry and wet ends of the full soil moisture range, soil moisture in West and East is only slightly skewed. There are some strayed data points in Utah that have negative skewness, even though the means are less than its mid-range soil moisture. This is associated with the relative uniform soil moisture conditions in June that made the statistics less representative. AMSR-E soil moisture, on the other hand, shows all positive skewness in each region, which is caused by its drier than its mid-range spatial means in all regions.

\subsection{Impact of sampling density on spatial mean and spatial variability}

Due to the limitation of the SCAN network, the above statistical analyses were based on scattered data points that may not represent the true averaged behavior of soil moisture in each region. To evaluate the impact of sampling sizes (number of sampling points within each region), daily mean and spatial variability of Noah soil moisture were calculated using all grid points (between $25^{\circ} \mathrm{N}$ and $49^{\circ} \mathrm{N}$ for all three regions and the east of $121^{\circ} \mathrm{W}$ for West to exclude the coastal area) and compared with those using data at SCAN locations only. The scatter plot of Fig. 6 (left panel) shows that daily means calculated using the two sampling schemes are nearly unbiased in each region. This is further confirmed by their temporally averaged mean values in Table 1 . This result suggests that the true spatial mean of Noah soil moisture in each region is sufficiently sampled by data (i.e., Noah soil moisture) at SCAN locations alone.

Similarly, the standard deviation (right panel) calculated from SCAN locations is in generally good agreement with that based on all grid points, except in West and MidCon where larger deviations are observed. Figure 7 shows that spatial variability of Noah soil moisture calculated from all grid points exhibits similar climate dependency as that in Fig. 3. With increased sample sizes, the impact of extents is more evident as the variability in West and East is noticeably larger than those in Utah and Miss-Tenn. The uneven spatial distribution of SCAN sites may be responsible for the lack of impact of scales on spatial variability as shown in Fig. 3. Table 1 shows that, on average, the spatial variability of soil moisture did not change significantly with increased sampling density in most regions, except in MidCon and West, suggesting that the spatial variability of Noah 

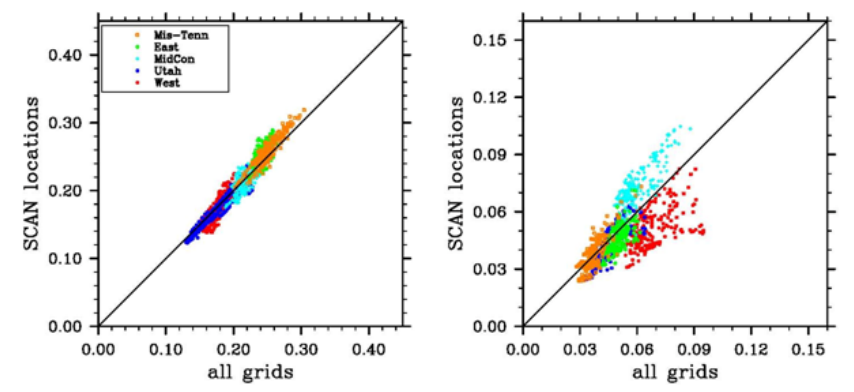

Fig. 6. Scatter plot of Noah daily mean soil moisture $\left(\mathrm{cm}^{3} \mathrm{~cm}^{-3}\right.$, left panel) and standard deviation (right panel) calculated at SCAN locations versus those over all grid points in each region.

estimates as sampled at SCAN locations is representative of Noah's true soil moisture spatial variability. The significant increase of spatial variability in West is likely associated with the increase in total precipitation (see Table 2) when all grid points were sampled. Increasing precipitation increased the wetness of soil moisture, which led to increased spatial variability because of the positive correlation between spatial variability and mean soil moisture in West. In MidCon, mean soil moisture slightly increased even though precipitation decreased with increased sampling, indicating that SCAN locations missed some low precipitation spots. This preferential sampling of the SCAN network may be the reason why spatial variability decreased with increased sampling in MidCon.

Because the support of SCAN soil moisture is smaller than that of Noah estimates, this test does not necessarily imply that the mean and spatial variability at the point scale are accurately captured by SCAN soil moisture. However, given the good correlations between SCAN and Noah soil moisture (Table 3) and the agreement between NLDAS and SCAN precipitation (Table 2), this test provides some confidence that statistics based on SCAN data are representative of the climate condition in each region.

\subsection{Factors contributing to soil moisture spatial variability}

Previous studies have linked factors such as atmospheric forcing, ET, soil texture, topographical features and vegetation types (see review by Rosenbaum et al., 2012) to soil moisture spatial variability and its relationship with mean soil moisture. For the scale of this study, physical processes such as precipitation and ET (which includes the effect of vegetation and radiation) are the major contributors to soil moisture spatial variability. Topography, which mainly affects soil moisture distribution at hillslope scales, does not play a significant role because SCAN sites are separated by much longer distances. Soil texture, which affects soil moisture through root zone uptake and drainage, also plays a lesser role than atmospheric forcing at large scales. For

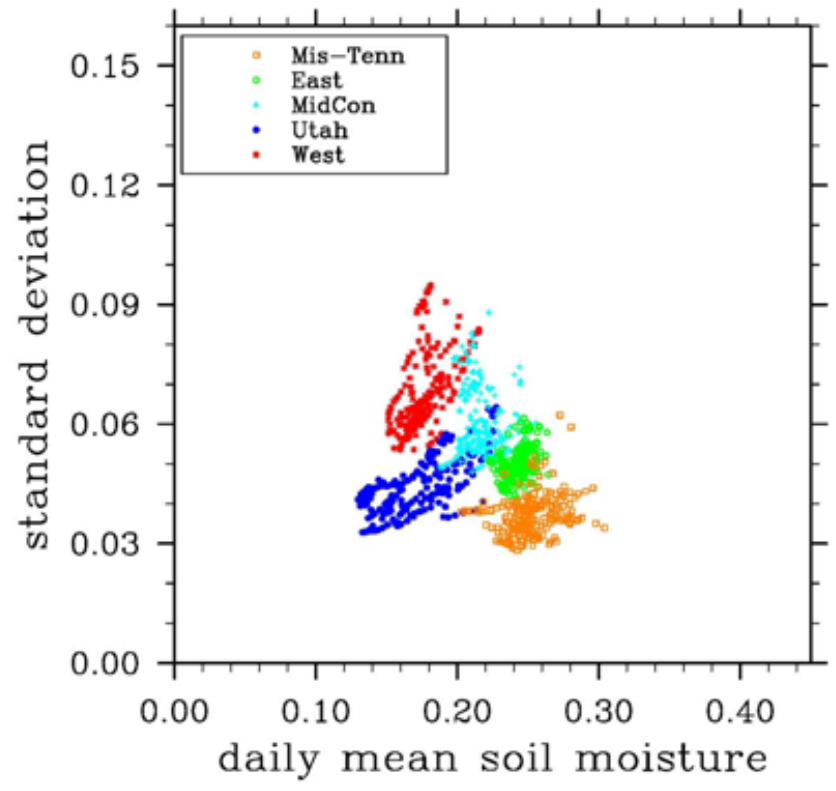

Fig. 7. Standard deviation of Noah soil moisture as a function of daily mean soil moisture $\left(\mathrm{cm}^{3} \mathrm{~cm}^{-3}\right)$. Statistics were calculated using all gridded data in each region.

instance, drainage occurring at a northwestern US location may differ from that at a southwestern US location due to soil texture, but the spatial variability of precipitation and ET at these two locations plays an even stronger role in their differences. Note that even though soil types observed at SCAN sites are limited (mainly silt loam and fine sandy loam), it does not mean that the spatial variability of soil moisture is significantly under-represented by SCAN data because their spatial distribution can capture a significant degree of variability in precipitation.

Figure 8 shows that precipitation spatial variability always positively correlates with mean precipitation, regardless of climate conditions. But the effect of precipitation on soil moisture spatial variability depends on soil water capacity in each climate. In dry climates where soils have large water capacity, soil moisture is responsive to changes in precipitation (see Fig. 4), i.e., a larger increase in precipitation leads to a larger increase in soil moisture. This strong correlation means that increased precipitation results in increased soil wetness which then leads to increased soil moisture spatial variability due to the positive correlation between precipitation variability and mean precipitation. Thus, precipitation alone can be responsible for the positive correlation between soil moisture spatial variability and mean soil moisture in the West and Utah. In addition, ET is constrained by moisture availability in a dry climate and is positively correlated with precipitation (Xia et al., 2012), meaning it also contributes to the positive correlation in the same way as precipitation.

The relationship between precipitation and soil moisture becomes more complex in wet climates due to reduced 

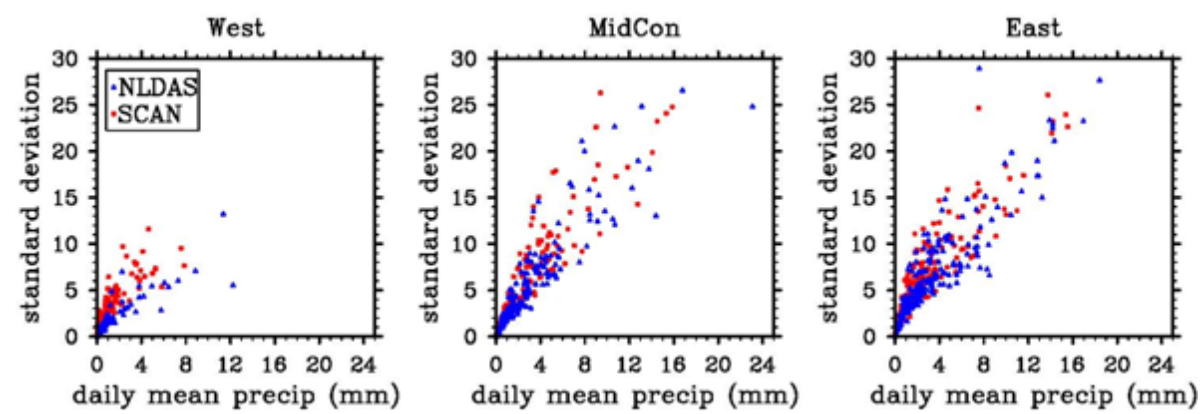

Fig. 8. Spatial variability (standard deviation) of daily precipitation ( $\mathrm{mm}$ ) as a function of mean precipitation. Daily precipitation of NLDAS and SCAN were first calculated at each SCAN location and then used to calculate spatial mean and standard deviation in each region.

available soil water capacity. With increased precipitation, more runoff is generated and so not all precipitation spatial variability is transferred to soil moisture. Impacts of ET on soil moisture also decrease because ET is now limited by energy availability. Rosenbaum et al. (2012) used the reduced impact from ET to explain the negative correlation between spatial variability and mean soil moisture. As soil becomes even wetter, soil moisture approaches its upper limit, saturation, where a minimum spatial variability should be reached because a bounded distribution normally exhibits minimum variances at the boundaries (Western et al., 2003). In the case of soil moisture, spatial variability at its two bounds should equal the spatial variability of wilting point and porosity (Western et al., 2003; Famiglietti et al., 2008). Indeed, spatial variability of Noah soil moisture is larger than the spatial variability of wilting points (not shown) used in the model in the drier range of soil moisture and larger than the spatial variability of porosity in the wetter range.

From a physical point of view, boundedness causes related physical processes to lose their interactions with soil moisture at the wilting point and saturation; as a result, the maximum spatial variability should occur around the mid-range soil moisture where boundedness has the weakest impact. Consequently, the positive and negative correlation should co-exist if spatial means extend to both the drier and wetter side of the soil moisture range as seen in SCAN soil moisture. However, the degree of correlation and the peak value in the upward convexity, which reflect the strength of the interaction between physical processes and soil moisture, should depend on the processes involved and their relative strength.

\subsection{Scale dependency of spatial variability}

To explore the scale dependency of soil moisture spatial variability and its relationship with climate, a range of extents shown in Fig. 1 (dark and light green concentric squares) were used to calculate the spatial variability of Noah and AMSR-E soil moisture. Since SCAN soil moisture only has one extent in MidCon, spatial variability of soil moisture by Famiglietti et al. (2008) at the $2.5 \mathrm{~m}, 16 \mathrm{~m}, 100 \mathrm{~m}$, $800 \mathrm{~m}, 1600 \mathrm{~m}$ and $50000 \mathrm{~m}$ extents (values taken from their
Fig. 11) were combined with SCAN spatial variability in MidCon. Most their measurements were obtained from the Great Plains, which has the similar climate condition as MidCon. To obtain a unique spatial variability value for each extent, the daily spatial variability of SCAN, Noah and AMSRE were averaged over the 198 days and plotted against extents in Fig. 9.

Log-transformation was used in Fig. 9 because, based on the self-similarity theory, the spatial variability is related to scales in an exponential function (the so-called power law), which can be linearized through log-transformation (Hu et al., 1998) as

$\log \left(\sigma_{\lambda}\right)=H \log (\lambda)+C$,

where $\lambda$ represents the scale (in this case the extent); $\sigma_{\lambda}$ is the spatial variability (standard deviation) at scale $\lambda ; C$ is a constant; $H$ is a scaling factor indicating the degree of dependency of spatial variability on scales. Following this relationship, linear relations were fitted for each data type (black lines in Fig. 9) and the slopes $(H)$ are given in Table 4.

The spatial variability of soil moisture increases as extents increase, with the increasing rate depending on data types and climate regions as shown in Table 4. From the smallest support (SCAN) to the largest (AMSR-E), the slope increases, meaning spatial variability increases faster as extents increase. This phenomenon can be explained by the diminished influence of supports on spatial variability with increasing extent. Specifically, the difference in supports will become negligible at large extents where spatial variability has reached such a high level that finer scale spatial variability becomes negligible. Because data sets with larger supports begin with lower spatial variability at smaller extents, their spatial variability has to increase at a faster rate to reach the same level of large scale spatial variability.

From the smallest (SCAN) to the largest support (AMSRE), soil moisture spatial variability decreases in each region. Note that the spatial variability of SCAN soil moisture in Fig. 9 was derived from climate regions and subregions, while the spatial variability of Noah and AMSR-E were obtained from the concentric squares (shown in Fig. 1). 

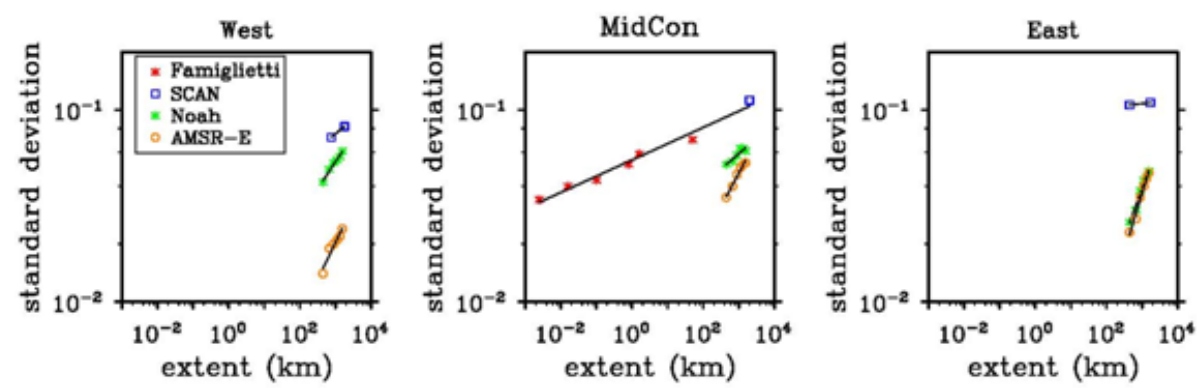

Fig. 9. Log-(standard deviation of soil moisture) as a function of log-(extent) in climate regions. Standard deviations of SCAN, Noah and AMSR-E soil moisture were obtained by temporally averaging daily values in each region over the 198 days.

Table 4. Slopes of linear relationship between log-(spatial variability of soil moisture and precipitation) and log-(extent) in each climate region. NLDAS precipitation $(\mathrm{mm})$ was calculated using values at SCAN locations only.

\begin{tabular}{lrrr}
\hline & West & MidCon & East \\
\hline In situ soil moisture & 0.15 & 0.085 & 0.02 \\
Noah soil moisture & 0.28 & 0.16 & 0.52 \\
AMSR-E soil moisture & 0.38 & 0.35 & 0.60 \\
NLDAS precipitation & 0.55 & 0.29 & 0.63 \\
\hline
\end{tabular}

However, the increased spatial variability with decreasing supports is true even when all statistics were calculated in the same region as shown in Table 1. In either case, the reduced spatial variability in AMSR-E and Noah (compared to that in SCAN) is mainly caused by reduced occurrences of extreme soil moisture values due to the use of spatially and temporally averaged forcing and parameter fields for Noah, and the large footprint of the sensor and maximum of two retrievals per day for AMSR-E. The spatial variability of AMSR-E becomes closer to that of Noah as the climate condition changes from dry to wet. This may indicate increased spatial correlation in wetter soil conditions which smoothes out the difference in spatial variability at the support of Noah and AMSRE. In theory, a linear relationship could also be fit for the log-(spatial variability) versus log-support which was not attempted due to an insufficient number of SCAN sites within each concentric square.

From the dry to wet climate, AMSR-E retrievals and Noah estimates exhibit the weakest scale dependency in MidCon and the strongest scale dependency in the East. To identify the source of such climate dependency for Noah soil moisture, the slope of NLDAS precipitation scale dependency is also provided in Table 4 . The spatial variability of NLDAS precipitation exhibits climate dependency that is similar to that of Noah soil moisture, confirming the strong influence of precipitation on soil moisture and its spatial variability as discussed in Sect. 4.3. One explanation for the weaker scale dependency of precipitation in MidCon is that the climate does not change as much as in West and East when the extent scale increases. For instance, with increasing extents in the West, precipitation differences between the wetter North and drier South become even larger, which leads to a larger (compared to that in MidCon) increase in precipitation spatial variability.

Figure 9 shows that data obtained from Famiglietti et al. (2008) and the SCAN data in MidCon can be fit by a linear function, suggesting that a single scaling relationship can potentially be used to scale spatial variability from very small scales to much larger scales. Brocca et al. (2012) reported a slope of 0.16 , using data collected in a similar climate condition as MidCon. Note that soil moisture data from Famiglietti et al. (2008) represent the top $6 \mathrm{~cm}$ of soil and were gathered from field campaigns conducted in different years (1997, 1999, 2002 and 2003), while SCAN soil moisture was measured hourly at $5 \mathrm{~cm}$ in 2008 . Using temporally averaged spatial variability values mitigated some of the impacts associated with sampling frequency and sampling periods since the dynamic range of spatial variability in June and July (sampling periods of Famiglietti et al., 2008) is very close to that of the entire period (Fig. 4). There was also no report of shallow groundwater, which can impact the vertical distribution of soil moisture (Rosenbaum et al., 2012), at the Famiglietti et al. (2008) and SCAN sites and so the behavior of soil moisture spatial variability at $5 \mathrm{~cm}$ is expected to be similar to that of the top $6 \mathrm{~cm}$ of soil. This apparent linear relationship should be further tested when more in situ soil moisture data become available.

\section{Summary and discussions}

We showed that in situ soil moisture exhibited distinct mean, spatial variability and skewness in each climate region. Further, the upward convex shape was observed for the relationship between spatial variability and mean soil moisture with positive and negative correlation for dry and wet climates, respectively, and no apparent correlation in the transition zone. Although this upward convex shape has been observed in much smaller scales, it has never been shown at continental scales and was achieved by merging statistics derived from 
contrasting climate zones where both the drier and wetter (compared to the mid-range soil moisture) ranges of mean soil moisture were reached. When mean soil moisture extends to both the drier and wetter ends of soil moisture, the upward convex shape results because minimum variability always occurs at the bounds for a bounded variable like soil moisture. Physical processes also influence soil moisture spatial variability and its relationship with mean soil moisture, with the maximum impacts occurring around the mid-range soil moisture where the effect of boundedness is the weakest.

Due to the limited number of SCAN sites in each region, the true mean and spatial variability may differ from that obtained from SCAN data. However, spatial means of SCAN soil moisture are consistent with the climate condition for each region, i.e., driest in West and wettest in East. In conjunction with the effect of boundedness, the upward convexity can still be expected when high spatial density data sets become available; but the degree of correlation in West/East and the maximum correlation in MidCon may change. Note that the correlation between spatial variability and mean soil moisture needs to be discussed with respect to the extent of measurements because the dynamic range of mean soil moisture may change with extents. For instance, the upward convexity can be observed from data collected in a sub-humid or humid region alone (e.g., Famiglietti et al., 2008; Rosenbaum et al., 2012) if spatial means reach to both the drier and wetter range of soil moisture.

Noah modeled soil moisture exhibited much smaller spatial variability than in situ soil moisture due to its larger support where temporally and spatially averaged forcing and parameter fields were used to derive the estimates. In addition, the upward convex relationship is barely discernible and skewness is nearly non-existent because their spatial means are close to the mid-range soil moisture in all regions. AMSR-E soil moisture retrievals, having the largest support, exhibited the smallest spatial variability among all three data types. The spatial variability of the retrievals showed only positive correlation with mean soil moisture, and skewness was also only positive. Despite differences in spatial means due to various reasons, the higher moments of AMSR-E and Noah showed dependencies on mean soil moisture that were similar to those of SCAN: positive correlation/skewness when most of their mean values (represented by the 25-75 percentiles in Fig. 2) were less than the midrange soil moisture of each data set, negative when most spatial means were greater than the mid-range soil moisture and no correlation when means are around or near the mid-range soil moisture. This dependency on mean soil moisture suggests that obtaining appropriate mean soil moisture at any given time (the box plot in Fig. 2 is a collection of all spatial means in 198 days) is critical for accurately modeling soil moisture spatial variability. Penn et al. (2009) showed that soil moisture spatial variability could be modeled correctly even without explicitly representing the topography, a major contributor to the spatial variability in their study site, if spatial mean soil moisture was appropriately simulated. One explanation for that result is that the boundedness of soil moisture largely dictates the dependency of spatial variability on mean soil moisture. Even though AMSR-E retrievals are generally less well validated by SCAN data, they exhibited smaller RMSE (assuming SCAN statistics are representative) than Noah soil moisture in West (Table 3) and may be used to improve the spatial mean of model estimates through data assimilation (Li et al., 2012). Data assimilation also provides a way to downscale retrievals in which high spatial and temporal resolution model estimates are dynamically merged with the retrievals.

Although observed and simulated soil moisture exhibited scalability as governed by the power law, their scale dependency differs: data with large supports showed stronger scale dependency than those with finer supports. The scale dependency also varies by climate regions: scale dependency of Noah and AMSR-E soil moisture was strongest in a wet climate and weakest in the transitional zone, which may be a consequence of the scale dependency of precipitation for Noah soil moisture. This relationship between the scale dependency of soil moisture and that of other physical processes has been suggested by Western et al. (2003) but has not been demonstrated in previous studies. One of the major differences of this study is that soil moisture spatial variability was dominated by precipitation variability, which is difficult to examine in small catchments where precipitation is relatively uniform and other controls (e.g., topography and soil texture) may have a stronger influence on soil moisture spatial variability. Future studies should be conducted on how the dependency of soil moisture spatial variability influences that of other processes such as ET and precipitation which would be useful for better understanding the interaction between the land and atmosphere and for improving the prediction of land surface processes. Based on the results presented here, such studies should be conducted in regions with intermediate precipitation such as MidCon where mean soil moisture is around the mid-range and processes can have the largest impact on soil moisture. This region of strong connections was also noted by Dirmeyer et al. (2009), who showed sustained interactions between soil moisture and precipitation in the Great Plains of the US through coupled landatmosphere modeling. Scalability of spatial variability from continental to catchment scales can be useful for obtaining spatial variability at intermediate scales for which appropriate measurement networks do not exist. Due to the limitations of Famiglietti et al. (2008) and SCAN data such as their differences in sampling depth and sampling period, further studies are needed to examine the scalability of soil moisture spatial variability across a wide range of extents; in particular, the impact of interannual variability of precipitation which is significant in MidCon on soil moisture spatial variability should be investigated.

The analyses described here were based on the full magnitude of soil moisture which contains the time-variant 
(anomalies) and time-invariant (mean) components. It is unknown if the spatial variability of soil moisture anomalies, which is a greater concern in some applications, exhibits similar climate dependency. Although Mittelbach and Seneviratne (2012) found that soil moisture anomalies only constituted a small percentage of soil moisture spatial variability, they also showed that the spatial variability in anomalies could differ from that in the full magnitude of soil moisture when precipitation changed. Information on the spatial variability of soil moisture anomalies is also critical for validating terrestrial water storage (TWS) products as provided by Gravity Recovery and Climate Experiment (GRACE, Tapley et al., 2004) satellites. GRACE TWS anomalies (deviations from the temporal mean) integrate anomalies in soil moisture, groundwater, snow and surface water, with soil moisture as one of the major components (e.g., Rodell et al., 2007). Thus, a similar study on the spatial variability of soil moisture anomalies and its scale dependency is also needed and will be conducted in the future when longer in situ data records become available to obtain more reliable mean soil moisture states in each region.

Acknowledgements. We would like to thank Heye Bogena and an anonymous reviewer for their comments and suggestions that have helped to improve the quality of this paper.

Edited by: H.-J. Hendricks Franssen

\section{References}

Brocca, L., Morbidelli, R., Melone, F., and Moramarco, T.: Soil moisture spatial variability in experimental areas of central Italy, J. Hydrol., 333, 356-373, doi:10.1016/j.jhydrol.2006.09.004, 2007

Brocca, L., Tullo, T., Melone, F., Moramarco, T., and Morbidelli, R.: Catchment scale soil moisture spatial-temporal variability, J. Hydrol., 422-423, 63-75, 2012.

Choi, M., Jacobs, J. M., and Cosh, M. H.: Scaled spatial variability of soil moisture fields, Geophys. Res. Lett., 34, L01401, doi:10.1029/2006GL028247, 2007.

Cosgrove, B., Lohmann, D., Mitchell, K., Houser, P., Wood, E. F., Schaake, J. C., Robock, A., Marshall, C., Sheffield, J., Duan, Q., Luo, L., Higgins, R. W., Pinker, R. T., Tarpley, J. D., and Meng, J.: Real-time and retrospective forcing in the North American Land Data Assimilation System (NLDAS) project, J. Geophys. Res., 108, 8842, doi:10.1029/2002JD003118, 2003.

Crow, W. and Wood, E.: Multi-scale dynamics of soil moisture variability observed during SGP'97, Geophys. Res. Lett., 26, 34853488, 1999.

Crow, W. and Wood, E.: The value of coarse-scale soil moisture observations for regional surface energy balance modeling, J. Hydrometeorol., 3, 467-482, 2002.

Dirmeyer, P., Schlosser, C. A., and Brubaker, K. L.: Precipitation, recycling, and land memory: an integrated analysis, J. Hydrometeorol., 10, 278-288, doi:10.1175/2008JHM1016.1, 2009.
Famiglietti, J. S., Devereaux, J. A., Laymon, C. A., Tsegaye, T., Houser, P. R., Jackson, T. J., Graham, S. T., Rodell, M., and van Oevelen, P. J.: Ground-based investigation of soil moisture variability within remote sensing footprints during the Southern Great Plains 1997 (SGP97) Hydrology Experiment, Water Resour. Res., 35, 1839-1851, 1999.

Famiglietti, J. S., Ryu, D., Berg, A. A., Rodell, M., and Jackson, T. J.: Field observations of soil moisture variability across scales, Water Resour. Res., 44, W01423, doi:10.1029/2006WR005804, 2008.

$\mathrm{Hu}, \mathrm{Z}$., Chen, Y., and Islam, S.: Multiscaling properties of soil moisture images and decomposition of large- and small-scale features using wavelet transforms, Int. J. Remote Sens., 19, 2451-2467, 1998.

Jackson, T. J.: Measuring surface soil moisture using passive microwave remote sensing, Hydrol. Process., 7, 139-152, 1993.

Li, B., Toll, D., Zhan, X., and Cosgrove, B.: Improving estimated soil moisture fields through assimilation of AMSR-E soil moisture retrievals with an ensemble Kalman filter and a mass conservation constraint, Hydrol. Earth Syst. Sci., 16, 105-119, doi:10.5194/hess-16-105-2012, 2012.

Mitchell, K. E., Lohmann, D., Houser, P. R., Wood, E. F., Schaake, J. C., Robock, A., Cosgrove, B. A., Sheffield, J., Duan, Q., Luo, L., Higgins, R. W., Pinker, R. T., Tarpley, J. D., Lettenmaier, D. P., Marshall, C. H., Entin, J. K., Pan, M., Shi, W., Koren, V., Meng, J., Ramsay, B. H., and Bailey, A. A.: The multi-institution North American Land Data Assimilation System (NLDAS): Utilizing multiple GCIP products and partners in a continental distributed hydrological modeling system, J. Geophys. Res., 109, D07S90, doi:10.1029/2003JD003823, 2004.

Mittelbach, H. and Seneviratne, S. I.: A new perspective on the spatio-temporal variability of soil moisture: temporal dynamics versus time-invariant contributions, Hydrol. Earth Syst. Sci., 16, 2169-2179, doi:10.5194/hess-16-2169-2012, 2012.

Njoku, E. G., Jackson, T. J., Lakshmi, V., Chan, T. K., and Nghiem, S. V.: Soil moisture retrieval from AMSR-E, IEEE T. Geosci. Remote, 41, 215-229, 2003.

Parada, L. M. and Liang, X.: A downscaling framework for L band radiobrightness temperature imagery, J. Geophys. Res., 108, 8863, doi:10.1029/2003JD003637, 2003.

Penna, D., Borga, M., Norbiato, D., and Fontana, G. D.: Hillslope scale soil moisture variability in a steep alpine terrain, J. Hydrol., 364, 311-327, 2009.

Rodriguez-Iturbe, I., Vogel, G. K., Rigon, R., Entekhabi, D., Castelli, F., and Rinaldo, A.: On the spatial organization of soil moisture fields, Geophys. Res. Lett., 106, 2757-2760, 1995.

Ryu, D. and Famiglietti, J. S.: Characterization of footprintscale surface soil moisture variability using Gaussian and beta distribution functions during the Southern Great Plains 1997 (SGP97) hydrology experiment, Water Resour. Res., 41, W12433, doi:10.1029/2004WR003835, 2005.

Rodell, M., Chen, J., Kato, H., Famiglietti, J., Nigro, J., and Wilson, C.: Estimating ground water storage changes in the Mississippi River basin (USA) using GRACE, Hydrogeol. J., 15, 159-166, doi:10.1007/s10040-006-0103-7, 2007.

Rosenbaum, U., Bogena, H., Herbst, M., Huisman, J. A., Peterson, T. J., Weuthen, A., and Vereecken, H.: Seasonal and event dynamics of spatial soil moisture patterns at the small catchment scale, Water Resour. Res., 48, W10544, 
doi:10.1029/2011WR011518, 2012.

Schaefer, G. L, Cosh, M. H., and Jackson, T. J.: The USDA Natural Resources Conservation Service Soil Climate Analysis Network (SCAN), J. Atmos. Ocean. Tech., 24, 2073-2077, 2007.

Seyfried, M. S., Grant, L. E., Du, E., and Humes, K.: Dielectric loss and calibration of the hydra probe soil water sensor, Vadose Zone J., 4, 1070-1079, 2005.

Tapley, B. D., Bettadpur, S., Ries, J. C., Thompson, P. F., Watkins, M. M.: GRACE measurements of mass variability in the Earth system, Science, 305, 503-505, 2004.

Wang, C., Zuo, Q., and Zhang, R.: Estimating the necessary sampling size of surface soil moisture at different scales using a random combination method, J. Hydrol., 352, 309-321, doi:10.1016/j.jhydrol.2008.01.011, 2008.

Western, A. W. and Blösch, G.: On the spatial scaling of soil moisture, J. Hydrol., 217, 203-224, 1999.
Western, A. W., Grayson, R. B., Blöschl, G., and Wilson, D. J.: Spatial variability of soil moisture and its implications for scaling, in: Scaling Methods in Soil Physics, editede by: Perchepsky, Y., Selim, M., and Radcliffe, A., CRC Press, Boca Raton, 119-142, 2003.

Xia, Y., Ek, M., Wei, H., and Meng, J.: Comparative analysis of relationships between NLDAS-2 forcings and model outputs, Hydrol. Process., 26, 467-474, doi:10.1002/hyp.8240, 2012.

Zhan, X., Liu, J., Jackson, T. J., Meng, J., Weng, F., and Mitchell, K.: Merging satellite soil moisture retrievals and model simulations for a blended consistent operational global soil moisture data product, SPIE Annual Conference on Atmospheric and Environmental Remote Sensing Data Processing and Utilization IV: Readiness for GEOSS II. San Diego, CA, 10-14 August, 2008. 